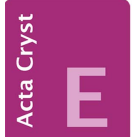
COMMUNICATIONS

ISSN 2056-9890

\section{Crystal structure of 2-\{[(5-nitrothiophen- 2-yl)methylidene]amino\} phenol}

\author{
Figen Koçak, ${ }^{\text {a Hasan Tanak, }}{ }^{\text {a* Erbil Ağar, }}{ }^{\mathrm{b}}$ Onur Erman \\ Doğan ${ }^{\mathrm{c}}$ and Namık Özdemir ${ }^{\mathrm{d}}$
}

${ }^{a}$ Department of Physics, Faculty of Arts \& Science, Amasya University, TR-05100 Amasya, Turkey, ${ }^{\mathbf{b}}$ Department of Chemistry, Faculty of Arts \& Science, Ondokuz

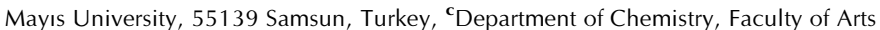
\& Science, Ondokuz Mayıs University, TR-55139 Kurupelit-Samsun, Turkey, and ${ }^{d}$ Department of Physics, Ondokuz Mayıs University, TR-55139 Samsun, Turkey.

*Correspondence e-mail: hasantanak@gmail.com

Received 9 May 2015; accepted 14 May 2015

Edited by W. T. A. Harrison, University of Aberdeen, Scotland

The title compound, $\mathrm{C}_{11} \mathrm{H}_{8} \mathrm{~N}_{2} \mathrm{O}_{3} \mathrm{~S}$, is roughly planar; the dihedral angle between the planes of the thiophene and benzene rings is $8.38(10)^{\circ}$. An intramolecular $\mathrm{O}-\mathrm{H} \cdots \mathrm{N}$ hydrogen bond generates an $S(5)$ ring motif. In the crystal, molecules are linked into centrosymmetric dimers by pairs of $\mathrm{O}-\mathrm{H} \cdots \mathrm{O}$ hydrogen bonds with an $R_{2}^{2}(22)$ graph-set motif. Aromatic $\pi-\pi$ stacking interactions [centroid-centroid separations $=3.653$ (3) and 3.852 (3) $\AA$ ] link the dimers into a threedimensional network.

Keywords: crystal structure; Schiff bases; phenol; hydrogen bonding; $\pi-\pi$ stacking.

CCDC reference: 1400935

\section{Related literature}

For Schiff bases as ligands, see: Aydoğan et al. (2001); Tanak et al. (2009). For related structures, see: Tanak et al. (2013, 2014).

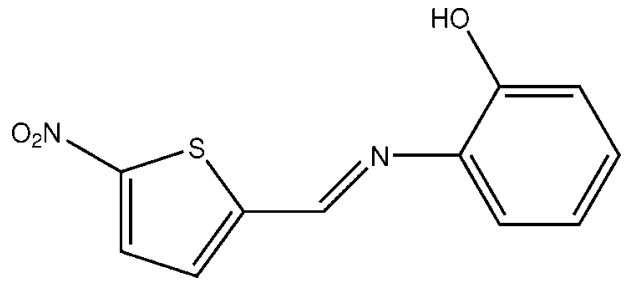

\section{Experimental}

2.1. Crystal data

$\mathrm{C}_{11} \mathrm{H}_{8} \mathrm{~N}_{2} \mathrm{O}_{3} \mathrm{~S}$

$M_{r}=248.25$

Monoclinic, $P 2_{1} / c$

$a=10.642(5) \mathrm{A}$

$b=7.043(5) \AA$

$c=14.535(5) \AA$

$\beta=93.566(5)^{\circ}$

$$
\begin{aligned}
& V=1087.3(10) \AA^{3} \\
& Z=4 \\
& \text { Mo } K \alpha \text { radiation } \\
& \mu=0.29 \mathrm{~mm}^{-1} \\
& T=293 \mathrm{~K} \\
& 0.68 \times 0.37 \times 0.15 \mathrm{~mm}
\end{aligned}
$$

\subsection{Data collection}

Stoe IPDS diffractometer Absorption correction: integration ( $X$-RED32; Stoe \& Cie, 2002)

$T_{\min }=0.877, T_{\max }=0.965$

7883 measured reflections 2254 independent reflections 1696 reflections with $I>2 \sigma(I)$ $R_{\text {int }}=0.114$

\subsection{Refinement}

$R\left[F^{2}>2 \sigma\left(F^{2}\right)\right]=0.064$

$w R\left(F^{2}\right)=0.100$

$S=0.98$

2254 reflections

186 parameters

$\mathrm{H}$ atoms treated by a mixture of independent and constrained refinement

$\Delta \rho_{\max }=0.89{\mathrm{e} \AA^{-3}}^{-3}$

$\Delta \rho_{\min }=-0.45$ e $\AA^{-3}$

Table 1

Hydrogen-bond geometry $\left(\AA,^{\circ}\right)$.

\begin{tabular}{lllll}
\hline$D-\mathrm{H} \cdots A$ & $D-\mathrm{H}$ & $\mathrm{H} \cdots A$ & $D \cdots A$ & $D-\mathrm{H} \cdots A$ \\
\hline $\mathrm{O} 3-\mathrm{H} 1 \cdots \mathrm{O}^{\mathrm{i}}$ & $0.78(3)$ & $2.54(3)$ & $3.192(3)$ & $141(3)$ \\
$\mathrm{O} 3-\mathrm{H} 1 \cdots \mathrm{N} 2$ & $0.78(3)$ & $2.23(3)$ & $2.711(2)$ & $121(3)$ \\
\hline
\end{tabular}

Symmetry code: (i) $-x+1,-y,-z$.

Data collection: $X$-AREA (Stoe \& Cie, 2002); cell refinement: $X$-AREA ; data reduction: $X$-RED32 (Stoe \& Cie, 2002); program(s) used to solve structure: SHELXS97 (Sheldrick, 2008); program(s) used to refine structure: SHELXL97 (Sheldrick, 2008); molecular graphics: ORTEP-3 for Windows (Farrugia, 2012); software used to prepare material for publication: WinGX (Farrugia, 2012).

\section{Acknowledgements}

This study was supported financially by the Research Center of Amasya University (Project No. FMB-BAP 15-091).

Supporting information for this paper is available from the IUCr electronic archives (Reference: HB7421).

\section{References}

Aydoğan, F., Öcal, N., Turgut, Z. \& Yolaçan, C. (2001). Bull. Korean Chem. Soc. 22, 476-480.

Farrugia, L. J. (2012). J. Appl. Cryst. 45, 849-854.

Sheldrick, G. M. (2008). Acta Cryst. A64, 112-122.

Stoe \& Cie (2002). $X$-AREA and X-RED32. Stoe \& Cie, Darmstadt, Germany. Tanak, H., Agar, A. A. \& Büyükgüngör, O. (2013). J. Mol. Struct. 1048, 41-50.

Tanak, H., Agar, A. A. \& Büyükgüngör, O. (2014). Spectrochim. Acta Part A, 118, 672-682.

Tanak, H., Erşahin, F., Ağar, E., Yavuz, M. \& Büyükgüngör, O. (2009). Acta Cryst. E65, o2291. 


\section{supporting information}

Acta Cryst. (2015). E71, o418 [doi:10.1107/S2056989015009202]

\section{Crystal structure of 2-\{[(5-nitrothiophen-2-yl)methylidene]amino\}phenol}

\section{Figen Koçak, Hasan Tanak, Erbil Ağar, Onur Erman Doğan and Namık Özdemir}

\section{S1. Comment}

Schiff bases have long been employed as ligands for the complexation of metal ions (Aydoğan et al., 2001; Tanak et al., 2009).

In the title compound (Fig. 1), the molecular structure is almost planar. The dihedral angle between the $\mathrm{C} 1-\mathrm{C} 4 / \mathrm{S} 1$ thiophene and the $\mathrm{C} 6-\mathrm{C} 11$ phenyl rings is $8.38(10)^{\circ}$. The imino group is coplanar with the nitrothiophene ring as it can be shown by the $\mathrm{C} 3-\mathrm{C} 4-\mathrm{C} 5-\mathrm{N} 2$ torsion angle is 178.60 (19) ${ }^{\circ}$. The length of the $\mathrm{C} 5=\mathrm{N} 2$ double bond is 1.264 (2) $\AA$, it is slightly shorter than standart $1.28 \AA$ value of $\mathrm{C}=\mathrm{N}$ double bond and consistent with the related stuructures (Tanak et al., 2013; Tanak et al., 2014). The $\mathrm{C} 1-\mathrm{S} 1$ and $\mathrm{C} 4-\mathrm{S} 1$ bond lengths of the thiophene ring are slightly different than the accepted value for an $\mathrm{Cs} p^{2}-\mathrm{S}$ single bond (1.76 $\AA$ ), resulting from the conjugation of the electrons of atom $\mathrm{S} 1$ with atoms C1 and C4 (Tanak et al., 2014).

The crystal structure is stabilized by $\mathrm{O}-\mathrm{H} \cdots \mathrm{N}$ and $\mathrm{O}-\mathrm{H} \cdots \mathrm{O}$ type intra and intermolecular hydrogen bonds. An intramolecular $\mathrm{O} 3-\mathrm{H} 1 \cdots \mathrm{N} 2$ interaction (Table 1 and Fig. 1) generates an $\mathrm{S}(5)$ ring motif, 1995). In the crystal structure, pairs of $\mathrm{O} 3-\mathrm{H} 1 \cdots \mathrm{O} 2$ hydrogen bond link the molecules to form inversion dimer (Fig. 2) with an $R_{2}{ }^{2}(22)$ ring motif. The crystal structure also feaures $\pi-\pi$ stacking interactions with distances of $C g 1 \cdots C g 2=3.653$ (3) $\AA$ [symmetry code $=1$ $x,-1 / 2+y, 1 / 2-z$ ] and $C g 1 \cdots C g 2=3.852$ (3) $\AA$ [symmetry code $=1-x, 1 / 2+y, 1 / 2-z$ ], where $C g 1$ and $C g 2$ are the centroids of $\mathrm{C} 1-\mathrm{C} 4 / \mathrm{S} 1$ and $\mathrm{C} 6-\mathrm{C} 11$ rings, respectively. The details of the hydrogen bonds are summarized in Table 1. A packing diagram of the title compound is shown in Fig. 3.

\section{S2. Experimental}

The title compound was prepared by refluxing a mixture of a solution containing 5-nitrothiophene-2-carbaldehyde (18.4 $\mathrm{mg}, 0.117 \mathrm{mmol})$ in ethanol $(20 \mathrm{ml})$ and a solution containing 2-aminophenol (12.8 $\mathrm{mg}, 0.117 \mathrm{mmol})$ in ethanol $(20 \mathrm{ml})$. The reaction mixture was stirred for $5 \mathrm{~h}$ under reflux. Single crystals of the title compound for X-ray analysis were obtained by slow evaporation of an ethanol solution (yield 60\%; m.p. 430-432 K).

\section{S3. Refinement}

C-bound $\mathrm{H}$ atoms were positioned geometrically and refined using a riding model, with $\mathrm{C}-\mathrm{H}=0.93 \AA$ and $U_{\text {iso }}(\mathrm{H})=$ $1.2 U_{\mathrm{eq}}(\mathrm{C})$. The position of the $\mathrm{H} 1$ atom was obtained from a difference map of the electron density in the unit-cell and was refined freely. 


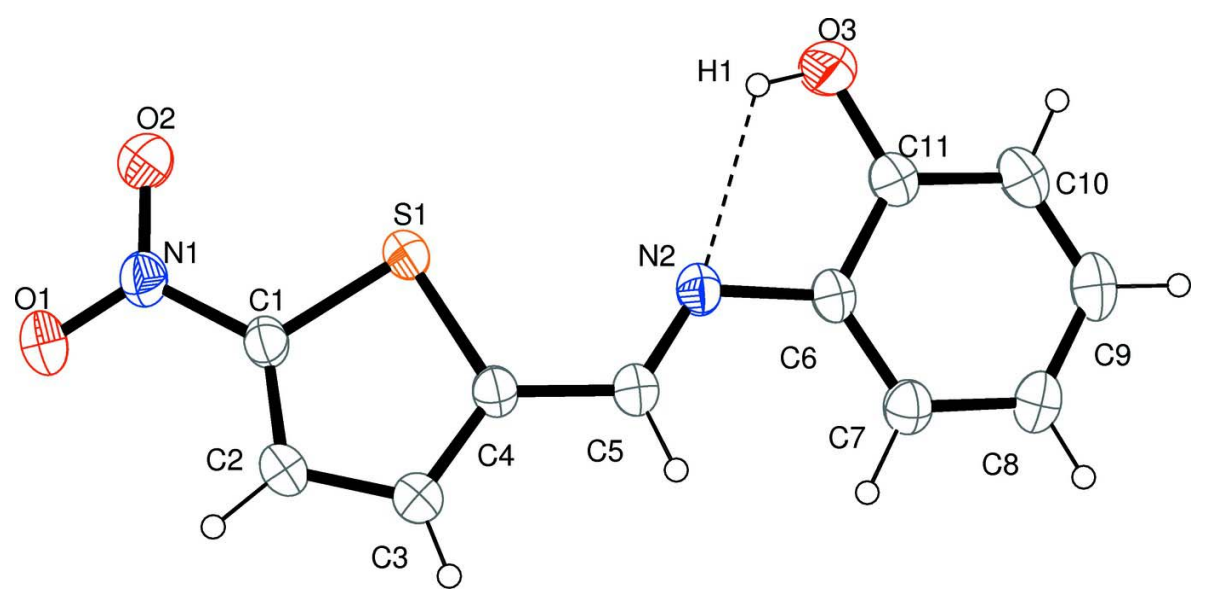

\section{Figure 1}

The molecular structure of the title compound, showing 30\% probability diplacement ellipsoids.

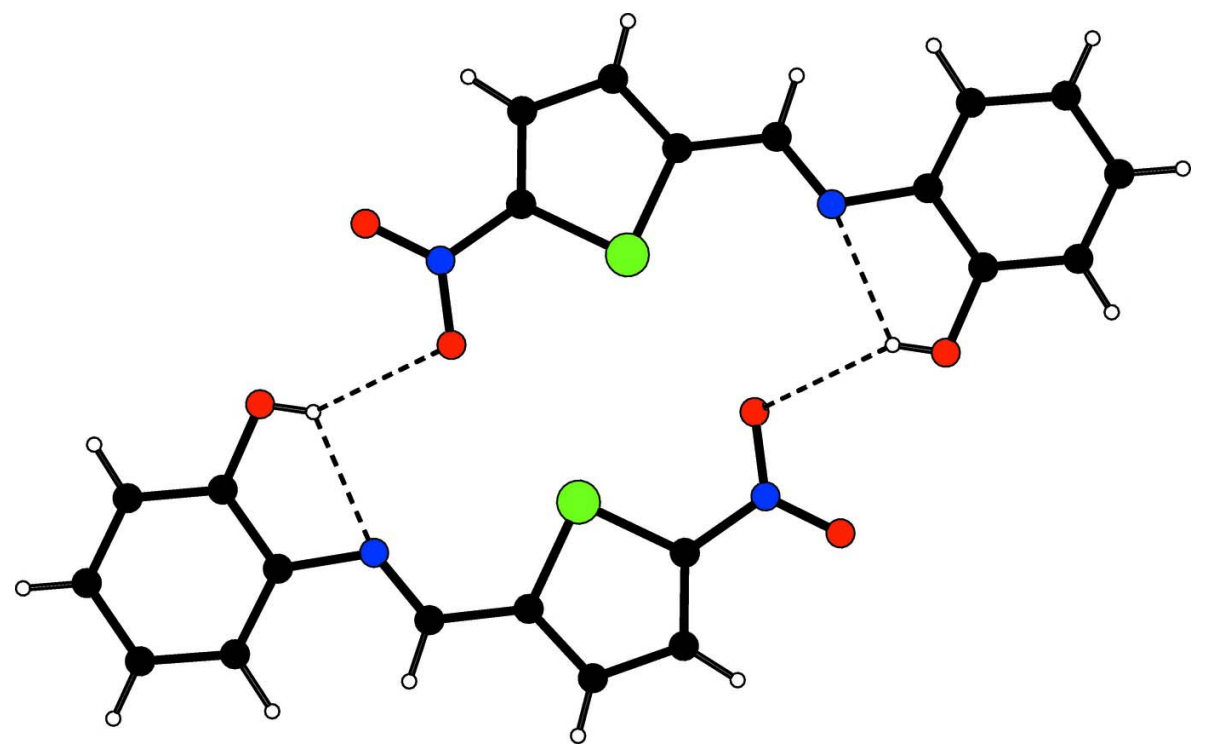

Figure 2

Centrosymmetric dimer with a central $R_{2}^{2}(22)$ ring motif. Dashed lines indicate hydrogen bonds. 


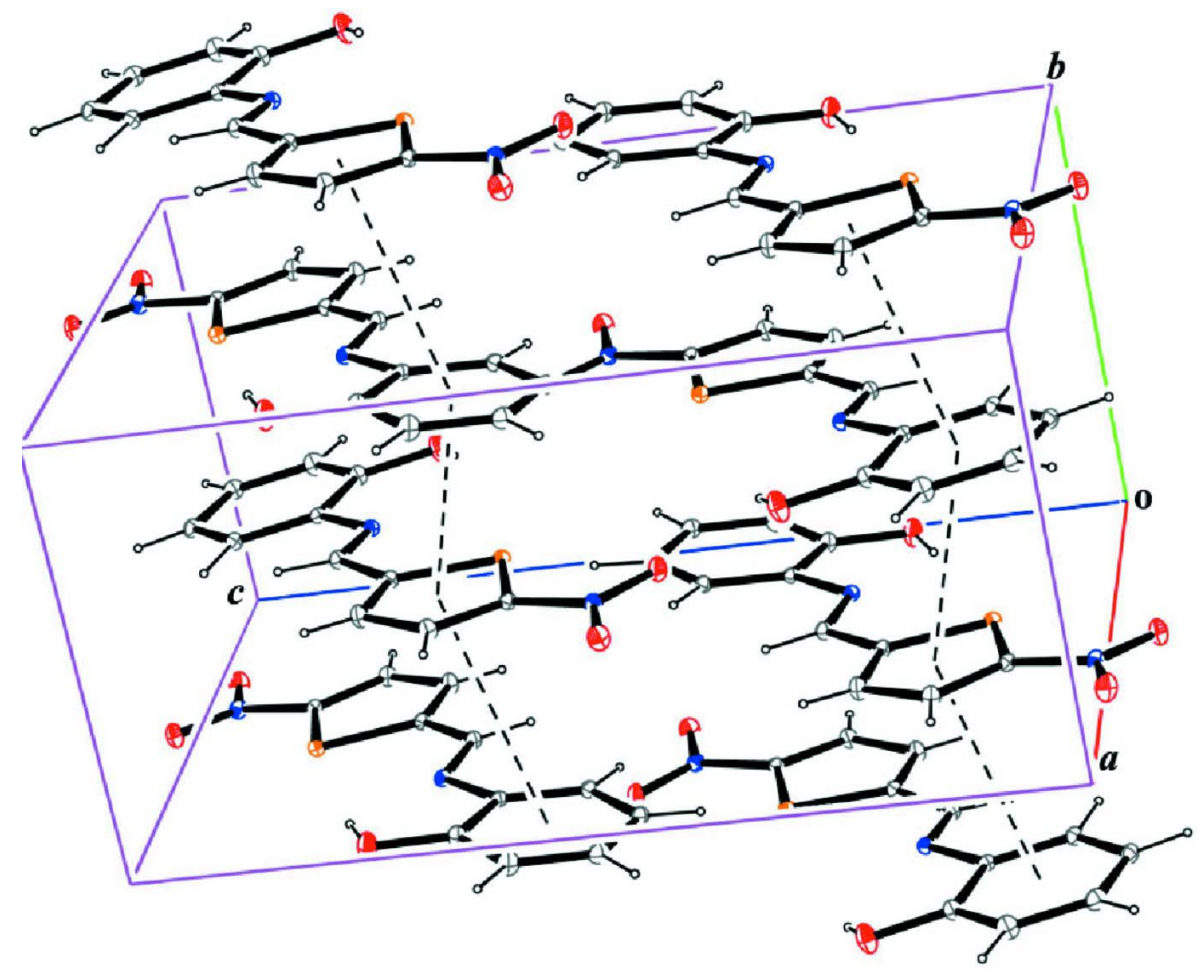

Figure 3

Packing diagram of the title compound.

\section{2-\{[(5-Nitrothiophen-2-yl)methylidene]amino\}phenol}

Crystal data

$\mathrm{C}_{11} \mathrm{H}_{8} \mathrm{~N}_{2} \mathrm{O}_{3} \mathrm{~S}$

$M_{r}=248.25$

Monoclinic, $P 2_{1} / c$

Hall symbol: -P $2 \mathrm{ybc}$

$a=10.642(5) \AA$

$b=7.043(5) \AA$

$c=14.535(5) \AA$

$\beta=93.566(5)^{\circ}$

$V=1087.3(10) \AA^{3}$

$Z=4$

\section{Data collection}

\section{Stoe IPDS}

\section{diffractometer}

Radiation source: fine-focus sealed tube

Graphite monochromator

Detector resolution: 6.67 pixels $\mathrm{mm}^{-1}$

rotation method scans

Absorption correction: integration

(X-RED32; Stoe \& Cie, 2002)

$T_{\min }=0.877, T_{\max }=0.965$
$F(000)=512$

$D_{\mathrm{x}}=1.516 \mathrm{Mg} \mathrm{m}^{-3}$

Mo $K \alpha$ radiation, $\lambda=0.71069 \AA$

Cell parameters from 9881 reflections

$\theta=1.9-29.0^{\circ}$

$\mu=0.29 \mathrm{~mm}^{-1}$

$T=293 \mathrm{~K}$

Prism, dark brown

$0.68 \times 0.37 \times 0.15 \mathrm{~mm}$

7883 measured reflections

2254 independent reflections

1696 reflections with $I>2 \sigma(I)$

$R_{\text {int }}=0.114$

$\theta_{\text {max }}=26.5^{\circ}, \theta_{\min }=1.9^{\circ}$

$h=-13 \rightarrow 13$

$k=-8 \rightarrow 8$

$l=-18 \rightarrow 18$ 


\section{Refinement}

Refinement on $F^{2}$

Least-squares matrix: full

$R\left[F^{2}>2 \sigma\left(F^{2}\right)\right]=0.064$

$w R\left(F^{2}\right)=0.100$

$S=0.98$

2254 reflections

186 parameters

0 restraints

Primary atom site location: structure-invariant direct methods
Secondary atom site location: difference Fourier map

Hydrogen site location: inferred from neighbouring sites

$\mathrm{H}$ atoms treated by a mixture of independent and constrained refinement

$w=1 /\left[\sigma^{2}\left(F_{\mathrm{o}}^{2}\right)+(0.0675 P)^{2}\right]$ where $P=\left(F_{\mathrm{o}}^{2}+2 F_{\mathrm{c}}^{2}\right) / 3$

$(\Delta / \sigma)_{\max }<0.001$

$\Delta \rho_{\max }=0.89$ e $\AA^{-3}$

$\Delta \rho_{\min }=-0.45$ e $\AA^{-3}$

\section{Special details}

Experimental. 360 frames, detector distance $=80 \mathrm{~mm}$

Geometry. All e.s.d.'s (except the e.s.d. in the dihedral angle between two 1.s. planes) are estimated using the full covariance matrix. The cell e.s.d.'s are taken into account individually in the estimation of e.s.d.'s in distances, angles and torsion angles; correlations between e.s.d.'s in cell parameters are only used when they are defined by crystal symmetry. An approximate (isotropic) treatment of cell e.s.d.'s is used for estimating e.s.d.'s involving 1.s. planes.

Refinement. Refinement of $F^{2}$ against ALL reflections. The weighted $R$-factor $w R$ and goodness of fit $S$ are based on $F^{2}$, conventional $R$-factors $R$ are based on $F$, with $F$ set to zero for negative $F^{2}$. The threshold expression of $F^{2}>\sigma\left(F^{2}\right)$ is used only for calculating $R$-factors(gt) $e t c$. and is not relevant to the choice of reflections for refinement. $R$-factors based on $F^{2}$ are statistically about twice as large as those based on $F$, and $R$ - factors based on ALL data will be even larger.

Fractional atomic coordinates and isotropic or equivalent isotropic displacement parameters $\left(\hat{A}^{2}\right)$

\begin{tabular}{|c|c|c|c|c|}
\hline & $x$ & $y$ & $z$ & $U_{\text {iso }} * / U_{\text {eq }}$ \\
\hline $\mathrm{S} 1$ & $0.58190(4)$ & $0.11508(6)$ & 0.11107 (3) & 0.04605 (16) \\
\hline N2 & 0.41409 (13) & 0.10939 (19) & $0.27382(9)$ & 0.0449 (3) \\
\hline N1 & $0.77113(14)$ & $0.1231(2)$ & $-0.00532(10)$ & $0.0532(4)$ \\
\hline $\mathrm{C} 1$ & $0.73710(15)$ & $0.1181(2)$ & $0.08813(11)$ & $0.0453(4)$ \\
\hline $\mathrm{C} 4$ & $0.62428(15)$ & $0.1069(2)$ & $0.22648(11)$ & $0.0460(4)$ \\
\hline C6 & $0.32806(15)$ & $0.1149(2)$ & $0.34380(11)$ & $0.0438(4)$ \\
\hline $\mathrm{O} 2$ & $0.68543(14)$ & $0.1159(2)$ & $-0.06611(9)$ & $0.0733(4)$ \\
\hline $\mathrm{C} 5$ & $0.53109(16)$ & 0.1040 & $0.29481(12)$ & $0.0496(4)$ \\
\hline $\mathrm{C} 7$ & $0.35846(18)$ & $0.1360(3)$ & $0.43745(12)$ & $0.0512(4)$ \\
\hline $\mathrm{C} 3$ & $0.75216(17)$ & 0.1053 & $0.24363(13)$ & $0.0625(5)$ \\
\hline O1 & $0.88152(13)$ & $0.1329(3)$ & $-0.02073(10)$ & $0.0807(5)$ \\
\hline $\mathrm{C} 2$ & $0.81816(17)$ & $0.1119(3)$ & $0.16344(13)$ & $0.0581(5)$ \\
\hline $\mathrm{O} 3$ & $0.16507(15)$ & 0.0835 & $0.22346(11)$ & $0.0904(6)$ \\
\hline $\mathrm{C} 8$ & 0.26579 (19) & 0.1470 & $0.49960(13)$ & $0.0574(5)$ \\
\hline $\mathrm{C} 11$ & $0.20103(17)$ & $0.1045(3)$ & $0.31379(13)$ & $0.0584(5)$ \\
\hline C9 & $0.1415(2)$ & $0.1387(3)$ & $0.46888(15)$ & $0.0665(5)$ \\
\hline $\mathrm{C} 10$ & $0.1095(2)$ & $0.1179(4)$ & $0.37699(17)$ & $0.0748(6)$ \\
\hline $\mathrm{H} 2$ & $0.904(2)$ & $0.113(3)$ & $0.1586(14)$ & $0.068(6)^{*}$ \\
\hline H3 & $0.787(2)$ & 0.107 (3) & $0.2986(17)$ & $0.073(7)^{*}$ \\
\hline H5 & $0.564(2)$ & $0.096(3)$ & $0.3560(17)$ & $0.079(7)^{*}$ \\
\hline H7 & $0.447(2)$ & $0.150(3)$ & $0.4606(13)$ & $0.063(6)^{*}$ \\
\hline $\mathrm{H} 10$ & $0.028(3)$ & $0.112(4)$ & 0.3543 (19) & $0.100(9)^{*}$ \\
\hline H9 & $0.080(2)$ & $0.151(3)$ & $0.5109(16)$ & $0.078(7)^{*}$ \\
\hline
\end{tabular}


supporting information

\begin{tabular}{|c|c|c|c|c|}
\hline $\mathrm{H} 8$ & $0.288(2)$ & $0.167(3)$ & $0.5609(16)$ & $0.069(6)^{*}$ \\
\hline H1 & $0.227(3)$ & $0.069(4)$ & $0.198(2)$ & $0.097(10)^{*}$ \\
\hline
\end{tabular}

Atomic displacement parameters $\left(\AA^{2}\right)$

\begin{tabular}{lllllll}
\hline & $U^{11}$ & $U^{22}$ & $U^{33}$ & $U^{12}$ & $U^{13}$ & $U^{23}$ \\
\hline S1 & $0.0373(2)$ & $0.0531(3)$ & $0.0481(2)$ & $-0.00158(18)$ & $0.00516(15)$ & $0.00117(19)$ \\
N2 & $0.0434(7)$ & $0.0456(8)$ & $0.0468(7)$ & $-0.0009(6)$ & $0.0106(5)$ & $-0.0022(6)$ \\
N1 & $0.0513(8)$ & $0.0589(9)$ & $0.0506(8)$ & $-0.0077(7)$ & $0.0128(6)$ & $0.0009(7)$ \\
C1 & $0.0413(8)$ & $0.0481(9)$ & $0.0475(8)$ & $-0.0043(7)$ & $0.0102(6)$ & $-0.0017(7)$ \\
C4 & $0.0415(8)$ & $0.0509(10)$ & $0.0463(8)$ & $-0.0008(7)$ & $0.0083(6)$ & $-0.0016(7)$ \\
C6 & $0.0416(8)$ & $0.0412(8)$ & $0.0496(8)$ & $0.0020(7)$ & $0.0113(6)$ & $0.0011(7)$ \\
O2 & $0.0625(8)$ & $0.1093(12)$ & $0.0482(7)$ & $-0.0094(8)$ & $0.0035(6)$ & $0.0076(7)$ \\
C5 & $0.0433(9)$ & $0.0598(11)$ & $0.0463(9)$ & $0.0000(8)$ & $0.0086(7)$ & $-0.0012(8)$ \\
C7 & $0.0498(9)$ & $0.0535(11)$ & $0.0510(9)$ & $0.0034(8)$ & $0.0087(7)$ & $0.0005(8)$ \\
C3 & $0.0435(9)$ & $0.0957(16)$ & $0.0482(10)$ & $-0.0015(9)$ & $0.0028(7)$ & $-0.0021(10)$ \\
O1 & $0.0533(8)$ & $0.1232(14)$ & $0.0681(9)$ & $-0.0151(8)$ & $0.0234(7)$ & $-0.0032(9)$ \\
C2 & $0.0368(8)$ & $0.0814(13)$ & $0.0567(10)$ & $-0.0030(9)$ & $0.0072(7)$ & $-0.0037(9)$ \\
O3 & $0.0490(8)$ & $0.160(2)$ & $0.0621(9)$ & $0.0045(10)$ & $0.0003(7)$ & $-0.0177(10)$ \\
C8 & $0.0652(11)$ & $0.0581(12)$ & $0.0506(10)$ & $0.0055(9)$ & $0.0174(9)$ & $0.0026(8)$ \\
C11 & $0.0448(9)$ & $0.0739(13)$ & $0.0572(10)$ & $-0.0007(9)$ & $0.0077(7)$ & $-0.0046(9)$ \\
C9 & $0.0600(11)$ & $0.0721(14)$ & $0.0707(13)$ & $0.0035(10)$ & $0.0295(10)$ & $0.0010(10)$ \\
C10 & $0.0410(10)$ & $0.1066(19)$ & $0.0783(14)$ & $-0.0021(11)$ & $0.0147(9)$ & $-0.0058(13)$ \\
& & & & & &
\end{tabular}

Geometric parameters $\left(A,{ }^{\circ}\right)$

\begin{tabular}{llll}
\hline $\mathrm{S} 1-\mathrm{C} 1$ & $1.7054(18)$ & $\mathrm{C} 7-\mathrm{C} 8$ & $1.380(3)$ \\
$\mathrm{S} 1-\mathrm{C} 4$ & $1.7107(18)$ & $\mathrm{C} 7-\mathrm{H} 7$ & $0.99(2)$ \\
$\mathrm{N} 2-\mathrm{C} 5$ & $1.264(2)$ & $\mathrm{C} 3-\mathrm{C} 2$ & $1.399(3)$ \\
$\mathrm{N} 2-\mathrm{C} 6$ & $1.411(2)$ & $\mathrm{C} 3-\mathrm{H} 3$ & $0.86(2)$ \\
$\mathrm{N} 1-\mathrm{O} 1$ & $1.212(2)$ & $\mathrm{C} 2-\mathrm{H} 2$ & $0.92(2)$ \\
$\mathrm{N} 1-\mathrm{O} 2$ & $1.231(2)$ & $\mathrm{O} 3-\mathrm{C} 11$ & $1.353(2)$ \\
$\mathrm{N} 1-\mathrm{C} 1$ & $1.428(2)$ & $\mathrm{O} 3-\mathrm{H} 1$ & $0.78(3)$ \\
$\mathrm{C} 1-\mathrm{C} 2$ & $1.352(3)$ & $\mathrm{C} 8-\mathrm{C} 9$ & $1.370(3)$ \\
$\mathrm{C} 4-\mathrm{C} 3$ & $1.368(3)$ & $\mathrm{C} 8-\mathrm{H} 8$ & $0.92(2)$ \\
$\mathrm{C} 4-\mathrm{C} 5$ & $1.447(2)$ & $\mathrm{C} 11-\mathrm{C} 10$ & $1.383(3)$ \\
$\mathrm{C} 6-\mathrm{C} 7$ & $1.387(3)$ & $\mathrm{C} 9-\mathrm{C} 10$ & $1.366(3)$ \\
$\mathrm{C} 6-\mathrm{C} 11$ & $1.396(2)$ & $\mathrm{C} 9-\mathrm{H} 9$ & $0.93(2)$ \\
$\mathrm{C} 5-\mathrm{H} 5$ & $0.94(2)$ & $\mathrm{C} 10-\mathrm{H} 10$ & $0.91(3)$ \\
& & & $113.16(17)$ \\
$\mathrm{C} 1-\mathrm{S} 1-\mathrm{C} 4$ & & $\mathrm{C} 4-\mathrm{C} 3-\mathrm{C} 2$ & $122.7(16)$ \\
$\mathrm{C} 5-\mathrm{N} 2-\mathrm{C} 6$ & $89.58(8)$ & $\mathrm{C} 4-\mathrm{C} 3-\mathrm{H} 3$ & $124.1(16)$ \\
$\mathrm{O} 1-\mathrm{N} 1-\mathrm{O} 2$ & $120.03(15)$ & $\mathrm{C} 2-\mathrm{C} 3-\mathrm{H} 3$ & $110.35(16)$ \\
$\mathrm{O} 1-\mathrm{N} 1-\mathrm{C} 1$ & $123.60(15)$ & $\mathrm{C} 1-\mathrm{C} 2-\mathrm{C} 3$ & $121.6(13)$ \\
$\mathrm{O} 2-\mathrm{N} 1-\mathrm{C} 1$ & $118.92(15)$ & $\mathrm{C} 1-\mathrm{C} 2-\mathrm{H} 2$ & $128.0(13)$ \\
$\mathrm{C} 2-\mathrm{C} 1-\mathrm{N} 1$ & $117.48(15)$ & $\mathrm{C} 3-\mathrm{C} 2-\mathrm{H} 2$ & $106(2)$ \\
$\mathrm{C} 2-\mathrm{C} 1-\mathrm{S} 1$ & $125.76(16)$ & $\mathrm{C} 9-\mathrm{C} 8-\mathrm{H} 3-\mathrm{H} 7$ & $119.92(19)$ \\
$\mathrm{N} 1-\mathrm{C} 1-\mathrm{S} 1$ & $114.73(13)$ &
\end{tabular}




\begin{tabular}{|c|c|c|c|}
\hline $\mathrm{C} 3-\mathrm{C} 4-\mathrm{C} 5$ & $126.26(16)$ & $\mathrm{C} 9-\mathrm{C} 8-\mathrm{H} 8$ & $120.7(14)$ \\
\hline $\mathrm{C} 3-\mathrm{C} 4-\mathrm{S} 1$ & $112.18(13)$ & $\mathrm{C} 7-\mathrm{C} 8-\mathrm{H} 8$ & $119.3(14)$ \\
\hline $\mathrm{C} 5-\mathrm{C} 4-\mathrm{S} 1$ & $121.56(13)$ & $\mathrm{O} 3-\mathrm{C} 11-\mathrm{C} 10$ & $118.94(18)$ \\
\hline $\mathrm{C} 7-\mathrm{C} 6-\mathrm{C} 11$ & $118.31(16)$ & $\mathrm{O} 3-\mathrm{C} 11-\mathrm{C} 6$ & $121.26(17)$ \\
\hline $\mathrm{C} 7-\mathrm{C} 6-\mathrm{N} 2$ & $126.03(16)$ & $\mathrm{C} 10-\mathrm{C} 11-\mathrm{C} 6$ & $119.80(18)$ \\
\hline $\mathrm{C} 11-\mathrm{C} 6-\mathrm{N} 2$ & $115.62(15)$ & $\mathrm{C} 10-\mathrm{C} 9-\mathrm{C} 8$ & $120.01(18)$ \\
\hline $\mathrm{N} 2-\mathrm{C} 5-\mathrm{C} 4$ & $122.75(16)$ & $\mathrm{C} 10-\mathrm{C} 9-\mathrm{H} 9$ & $120.7(14)$ \\
\hline $\mathrm{N} 2-\mathrm{C} 5-\mathrm{H} 5$ & $122.4(15)$ & $\mathrm{C} 8-\mathrm{C} 9-\mathrm{H} 9$ & $119.3(14)$ \\
\hline $\mathrm{C} 4-\mathrm{C} 5-\mathrm{H} 5$ & $114.9(15)$ & $\mathrm{C} 9-\mathrm{C} 10-\mathrm{C} 11$ & $120.9(2)$ \\
\hline $\mathrm{C} 8-\mathrm{C} 7-\mathrm{C} 6$ & $121.03(18)$ & $\mathrm{C} 9-\mathrm{C} 10-\mathrm{H} 10$ & $122.2(18)$ \\
\hline $\mathrm{C} 8-\mathrm{C} 7-\mathrm{H} 7$ & $118.6(12)$ & $\mathrm{C} 11-\mathrm{C} 10-\mathrm{H} 10$ & $116.8(18)$ \\
\hline $\mathrm{C} 6-\mathrm{C} 7-\mathrm{H} 7$ & $120.3(12)$ & & \\
\hline $\mathrm{O} 1-\mathrm{N} 1-\mathrm{C} 1-\mathrm{C} 2$ & $-4.2(3)$ & $\mathrm{C} 5-\mathrm{C} 4-\mathrm{C} 3-\mathrm{C} 2$ & $-179.07(19)$ \\
\hline $\mathrm{O} 2-\mathrm{N} 1-\mathrm{C} 1-\mathrm{C} 2$ & $175.3(2)$ & $\mathrm{S} 1-\mathrm{C} 4-\mathrm{C} 3-\mathrm{C} 2$ & $0.4(2)$ \\
\hline $\mathrm{O} 1-\mathrm{N} 1-\mathrm{C} 1-\mathrm{S} 1$ & $177.06(14)$ & $\mathrm{N} 1-\mathrm{C} 1-\mathrm{C} 2-\mathrm{C} 3$ & $-179.06(17)$ \\
\hline $\mathrm{O} 2-\mathrm{N} 1-\mathrm{C} 1-\mathrm{S} 1$ & $-3.4(2)$ & $\mathrm{S} 1-\mathrm{C} 1-\mathrm{C} 2-\mathrm{C} 3$ & $-0.3(2)$ \\
\hline $\mathrm{C} 4-\mathrm{S} 1-\mathrm{C} 1-\mathrm{C} 2$ & $0.44(16)$ & $\mathrm{C} 4-\mathrm{C} 3-\mathrm{C} 2-\mathrm{C} 1$ & $0.0(3)$ \\
\hline $\mathrm{C} 4-\mathrm{S} 1-\mathrm{C} 1-\mathrm{N} 1$ & $179.27(14)$ & $\mathrm{C} 6-\mathrm{C} 7-\mathrm{C} 8-\mathrm{C} 9$ & $-0.6(3)$ \\
\hline $\mathrm{C} 1-\mathrm{S} 1-\mathrm{C} 4-\mathrm{C} 3$ & $-0.44(16)$ & $\mathrm{C} 7-\mathrm{C} 6-\mathrm{C} 11-\mathrm{O} 3$ & $-179.9(2)$ \\
\hline $\mathrm{C} 1-\mathrm{S} 1-\mathrm{C} 4-\mathrm{C} 5$ & $179.02(15)$ & $\mathrm{N} 2-\mathrm{C} 6-\mathrm{C} 11-\mathrm{O} 3$ & $2.3(3)$ \\
\hline $\mathrm{C} 5-\mathrm{N} 2-\mathrm{C} 6-\mathrm{C} 7$ & $7.5(3)$ & $\mathrm{C} 7-\mathrm{C} 6-\mathrm{C} 11-\mathrm{C} 10$ & $0.9(3)$ \\
\hline $\mathrm{C} 5-\mathrm{N} 2-\mathrm{C} 6-\mathrm{C} 11$ & $-174.84(17)$ & $\mathrm{N} 2-\mathrm{C} 6-\mathrm{C} 11-\mathrm{C} 10$ & $-176.96(19)$ \\
\hline $\mathrm{C} 6-\mathrm{N} 2-\mathrm{C} 5-\mathrm{C} 4$ & $-177.11(16)$ & $\mathrm{C} 7-\mathrm{C} 8-\mathrm{C} 9-\mathrm{C} 10$ & $0.6(3)$ \\
\hline $\mathrm{C} 3-\mathrm{C} 4-\mathrm{C} 5-\mathrm{N} 2$ & $178.60(19)$ & $\mathrm{C} 8-\mathrm{C} 9-\mathrm{C} 10-\mathrm{C} 11$ & $0.1(4)$ \\
\hline $\mathrm{S} 1-\mathrm{C} 4-\mathrm{C} 5-\mathrm{N} 2$ & $-0.8(3)$ & $\mathrm{O} 3-\mathrm{C} 11-\mathrm{C} 10-\mathrm{C} 9$ & $179.8(2)$ \\
\hline $\mathrm{C} 11-\mathrm{C} 6-\mathrm{C} 7-\mathrm{C} 8$ & $-0.1(3)$ & $\mathrm{C} 6-\mathrm{C} 11-\mathrm{C} 10-\mathrm{C} 9$ & $-0.9(4)$ \\
\hline $\mathrm{N} 2-\mathrm{C} 6-\mathrm{C} 7-\mathrm{C} 8$ & $177.44(17)$ & & \\
\hline
\end{tabular}

Hydrogen-bond geometry $\left(\AA,{ }^{\circ}\right)$

\begin{tabular}{lllll}
\hline$D-\mathrm{H} \cdots A$ & $D-\mathrm{H}$ & $\mathrm{H} \cdots A$ & $D \cdots A$ & $D-\mathrm{H}^{\cdots} A$ \\
\hline $\mathrm{O} 3-\mathrm{H} 1 \cdots \mathrm{O} 2^{\mathrm{i}}$ & $0.78(3)$ & $2.54(3)$ & $3.192(3)$ & $141(3)$ \\
$\mathrm{O} 3-\mathrm{H} 1 \cdots \mathrm{N} 2$ & $0.78(3)$ & $2.23(3)$ & $2.711(2)$ & $121(3)$ \\
\hline
\end{tabular}

Symmetry code: (i) $-x+1,-y,-z$. 